Published in Cruz, N.C., J.L. Redondo, M. Berenguel, J.D. Álvarez, A. Becerra-Terón, P.M. Ortigosa. High performance computing for the heliostat field layout evaluation. Journal of Supercomputing, published online: 16 March 2016, DOI 10.1007/s11227-016-1698-7. http://link.springer.com/article/10.1007\%2Fs11227-016-1698-7

This is the Initial version of the authors: 


\title{
High Performance Computing for the heliostat field layout evaluation|
}

\author{
N.C. Cruz $\ddagger$ and J.L. Redondo $\ddagger$ and M. \\ Berenguel $\ddagger$ and P.M. Ortigosa $\ddagger$ and A. \\ Becerra-Teron $\ddagger$
}

Received: date / Accepted: date

\begin{abstract}
In Solar Central Receiver Systems (SCRS), the heliostat field is generally the most important subsystem in terms of initial investment and energy losses. Therefore, heliostat field layout needs to be carefully designed and optimized when deploying this kind of power facilities. This optimization procedure can be focused on multiple and heterogeneous criteria depending on particular factors that lead to define different optimization problems based on specific objective functions. However, objective functions defined for this problem are, in general terms, computationally very expensive. This fact may make an exhaustive optimization process infeasible, specially depending on the available resources, and forces particular simplifications at some steps of the process. Fortunately, some of the objective functions defined can benefit from parallelization, even though this idea is not usually pointed out or discussed, and then become affordable in better conditions. As a proof of concept of this situation, in this paper, three different parallelization approaches of heliostat field optical efficiency computation are studied.
\end{abstract}

Keywords Heliostat field layout · Parallelization · Optimization

\section{Introduction}

Solar Central Receiver Systems (SCRS) are one of the most important and promising flagships of renewable energies. This sort of systems basically consist of a radiation receiver and a broad set of high-reflectance mirrors, known as 'heliostats', that reflect and concentrate solar radiation over the mentioned receiver. Some interesting additional and more extensive information of this kind of systems can be found in $[1,4,13]$ and in chapter 10 of [7].

This work has been funded by grants from the Spanish Ministry of Economy and Competitiveness (TIN2012-37483-C03-03), Junta de Andalucía (P10-TIC-6002, P11-TIC7176 and P12TIC301). Juana López Redondo is a fellow of the Spanish 'Ramón y Cajal' contract program, co-financed by the European Social Fund

$\ddagger$ University of Almería, Dpt. of Informatics

E-mail: ncalvocruz@gmail.com \{jlredondo,beren,ortigosa,abecerra\}@ual.es

Corresponding author: N.C. Cruz 
The receiver, which is at a certain height over the ground by placing it on a tower, is a complex device designed to transfer reflected incident energy into a working fluid that increases its temperature. Then, this fluid can be used in a traditional powersteam cycle to generate electrical energy. Besides, the extreme conditions obtained at the receiver also have scientific applications.

Heliostats are far from being simple mirrors too. In fact, they are equipped with complex control systems that make them track the apparent Sun position along the day in order to keep the incident flux reflected to the receiver as the maximum possible. Its reflecting surface is also a very well studied component which aims to maximize the redirected solar radiation towards the target receiver. This objective is usually achieved, specially in large surface heliostats, by adding some curvature to it as well as by decomposing it into sub-surfaces called 'facets' which are placed with some canting in the structure that forms the heliostat.

The set of heliostats, with their properties and particular distribution over the SCRS land, forms what is known as 'heliostat field layout'. This SCRS subsystem represents approximately the $50 \%$ of total initial investment and can generate up to $40 \%$ of energetic loss [8] in the power station cycle. Therefore, the heliostat field needs to be carefully studied and designed to get the maximum solar radiation concentrated over the receiver. This design process suppose solving complex optimization problems in which heliostats, depending on the SCRS latitude and terrain, on the receiver characteristics and on their own properties, are distributed over the available land trying to fulfill, as best as possible, a set of criteria.

This set of criteria leads to define complex objective functions to optimize (maximize or minimize) which can range from single-objective to multi-objective optimization problems depending on specific interests. Some of most common 'criteria' or objectives are optical efficiency [11] and investment cost (two principles combined in [16]).

Objective functions applied are generally multimodal ones whose structure and behavior are not completely known in order to perform an analytical optimization process. Furthermore, they are functions that can be defined on a wide range of dimensions whose evaluation is very time consuming, specially when problem size becomes larger and many heliostats have to be placed. Taking into consideration what have been said, multiple approximations are applied to objective functions like defining griding models based on a reduced set of variables that make an initial surface discretization $[11,12$, $16,7,15]$, avoiding some calculations in particular stages [2,11,12], selecting specific instants of study (when applicable) [11]etc. However, applying parallelization and high performance computing techniques to the implementation of objective functions does not seem to be an emphasized option.

In the next section, a common objective function, the yearly irradiance weighted efficiency of heliostat field, is defined in a simplified version as a proof of concept. Then, three parallel implementations have been proposed to take advantage of implicit parallelism of the selected objective function in section 3. After that, results of speedup are shown in section 4. Finally conclusions are commented on and future work is proposed in section 5 .

\section{Studied function}

The selected function is the yearly irradiance weighted efficiency described in [11]. It represents the heliostat field layout capabilities to concentrate solar radiation over the 
receiver along the year and also taking into account incident solar radiation (irradiance) of each moment as a weighting factor. This combined point of view makes it very interesting. It can be formulated as described below, where $I_{b}(t)$ is the instantaneous beam irradiance and $\eta$ is the instantaneous field optical efficiency [11]:

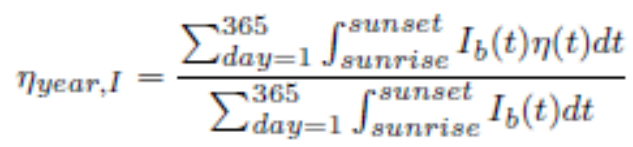

Field optical efficiency $\eta$, well detailed in chapter 10 of [7], is easily applicable for any number of heliostats by averaging each particular result. It can be defined by a chosen set of sub-factors that models different sources of energy loss in real heliostat fields. For our purpose, it is defined as in [11]

$$
\eta=\eta_{c o s} \cdot \eta_{s b} \cdot \eta_{i t c} \cdot \eta_{a a} \cdot \eta_{r e f}
$$

where $\eta_{c o s}, \eta_{s b}, \eta_{i t c}, \eta_{a a}$ and $\eta_{r e f}$ are the cosine, shadowing and blocking, interception, atmospheric attenuation and reflectivity efficiency respectively, which are described later in this section.

It is important to note that Eq.(2) can be also found based on other combinations of the underlying key concepts according to particular requirements. For instance, in [10], $\eta_{i t c}$ is not taken into direct account and $\eta_{s b}$ is just one factor (as in [11]) instead of being divided into $\eta_{\text {shadow }}$ and $\eta_{\text {block }}$ as in [7]. Furthermore, any of these terms could be removed, what would mean that would be supposed to be 1 (not an energy loss or negligible). The factor $I_{b}(t)$ of equation (1) is also abstractly defined so it is possible to use any available model to estimate direct solar radiation at any particular instant $t$.

Furthermore, it must also be taken into account that $\int_{\text {sunrise }}^{\text {sunset }} d t$ in Eq.(1) requires iterating over the day but it does not force to use a particular schema. For instance, in [11], some directives are given to optimize this process in a clever way: taking more time instants when Sun effect is more important. Finally, it is important to note that the common behavior of equation (1) is that it generally tends to 0 when the number of heliostats increases as distances and potential negative interactions between them are likely to increase.

\subsection{Sun position}

Heliostat field operation and configuration is directly linked to the Sun apparent movement along the year. Therefore, it is necessary to compute the Sun position as it is perceived from the power plant latitude at every instant $t$. There are several models such as the accurate PSA algorithm [3].

For this work, it has been selected the model explained in chapter 3 of [7]. According to it, the Sun apparent position (as azimuth $A$ and altitude $\alpha$ angles) can be obtained from solar declination $\delta$, latitude $\phi$ and hour angle $\omega$ by two expressions from chapter 3 of $[7]$.

$$
\begin{gathered}
\alpha=\sin ^{-1}(\sin \delta \sin \phi+\cos \delta \cos \omega \cos \phi) \\
A^{\prime}=\sin ^{-1}\left(\frac{-\cos \delta \sin \omega}{\cos \alpha}\right) ; \begin{array}{l}
A=180^{\circ}-A^{\prime} \text { if } \cos \omega \geq\left(\frac{\tan \delta}{\tan \phi}\right) \\
A=360^{\circ}+A^{\prime} \text { otherwhise }
\end{array}
\end{gathered}
$$




\subsection{Solar irradiance}

Heliostat field real performance is directly linked to the available solar radiation that falls on Earth along the day. Therefore, it is important to associate pure field configuration values with the amount of energy that can be really profited. It becomes capital when analyzing different SCRS power plants possible locations.

As occurs with Sun position, there are numerous models to estimate solar irradiance (the rate at which solar energy reaches a unit area at the Earth [7]) along the year with different accuracy and parameters as mentioned in [11]. However, for the sake of simplicity, the model used in this work for estimating solar beam normal radiation depends only on the 'air mass' $(A M)$ concept [7] and the location height above sea level. The equation for this model in $\left(\frac{\mathrm{kW}}{\mathrm{m}^{2}}\right)$ is [9]

$$
I_{b}=1.353 \cdot\left\{(1-a h) 0.7^{A M^{0.678}}+a h\right\}
$$

where $a=0.14$ and $h$ is the location height above the sea level in kilometers. It is important to note that 'AM' computation [7] depends on Sun altitude which at the same time varies along the day. Taking this into account, and as expressed in Eq.(1), $I_{b}$ also depends on time.

\subsection{Cosine efficiency}

Cosine efficiency is considered to be the most important energy loss source in heliostat fields. It is defined as the cosine of the angle formed by the solar beam direction and the reflective surface normal of the heliostat $[2,7]$. This factor depends on both solar apparent position and the location of every heliostat relative to the receiver. Cosine efficiency is directly linked to the percentage of its total reflection area that can redirect solar radiation towards the receiver properly as depicted in the figure 10.6 in chapter 10 of $[7]$.

In general terms, the cosine efficiency factor can be obtained from the dot product of solar beam direction and the heliostat surface normal according to the Law of Reflection $[2,11,7]$. In this paper, the defined context of operation (reference systems and general properties of elements) is the same as the one defined in [7] (clearly depicted in their figure 8.20 ). Taking this into account, this efficiency can be obtained from this equation $[7]$ :

$$
\cos 2 \theta_{i}=\frac{\left(z_{0}-z_{1}\right) \sin \alpha-e_{1} \cos \alpha \sin A-n_{1} \cos \alpha \cos A}{\sqrt{\left(z_{o}-z_{1}\right)^{2}+e_{1}^{2}+n_{1}^{2}}}
$$

where $\theta_{i}$ is the angle of incidence of central solar beam with the heliostat normal, $e_{1}, n_{1}$ and $z_{1}$ are the coordinates of the central point of the heliostat relative to the tower in East, North and Zenith respectively and $z_{0}$ is the reception point height over ground at the receiver.

\subsection{Shading and blocking efficiency}

SCRS power plants have several, even thousands, of heliostats distributed over their available land. This vast set of heliostats that share the same purpose combined with 
the fact that the most productive areas are known and preferable when distributing them make optical interferences a considerable energy loss. Then, every heliostat could cause or suffer from two problems: it could intercept part of the incident radiation that should reach any other heliostat generating then shadows over them, what is called 'shadowing', as well as it could also intercept part of the reflected radiation by any other heliostat in its path towards the receiver, what is called 'blocking'. These situations are perfectly depicted in figure 10.9 of [7]. It is also important to note that, depending on the situation, should be also considered as a shadowing source as done in [6].

Computing this factor is a complex and specially time consuming task. Therefore, there are numerous approximations that can be made to get acceptable results in a reasonable time, specially in control and optimization problems. Some of them are suppressing this factor in some stages of the whole computation process as in $[2,11,12]$ (it could even been fully suppressed when distribution layouts applied guarantee robustness to this problem), working over reduced pairs of potential blocking/shadowing heliostats and, avoiding realistic ray-tracing to specific approximations as in [6] and [11].

In this paper, the general approach described in [11] has been chosen with both discretization scheme and potentially blocking/shadowing heliostat selecting when projecting lines from every heliostat in the direction of the receiver (for blocking) and of the Sun (for shadowing). The applied equation for getting a numerical value in this context is the simple relation between neither blocked nor shadowed rays $n_{N}$ and the total studied number of them $n_{T}$ :

$$
\eta_{s b}=\frac{n_{N}}{n_{T}}
$$

\subsection{Interception efficiency}

Interception efficiency, also known as 'spillage factor' [2], consist on energy losses due to the lack of precision of heliostats while projecting solar beam irradiance over the receiver. This phenomenon, which depends on both heliostats and receiver properties [7], implies that part of the reflected radiation towards the receiver does not fall where expected.

In a similar way to shadowing and blocking computation, ray-tracing and light simulating approaches can be applied to determine this factor. However, analytical integration of the image shape produced over the receiver by heliostats using models as UNIZAR and HFLCAL is also a valid approach considering that ray-tracing should be avoided for optimization purposes [2].

The model implemented for this work calculates the interception efficiency a single time taking into account both heliostats and receiver dimensions as well as a general average sun shape error. It is the same as the implemented one in [5] (where further information is provided)

$$
\eta_{\text {int }}=\frac{p H\left(\frac{l w_{r}}{2 \sqrt{2} \sigma_{r}},-a_{r}, a_{r}\right) \cdot p H\left(\frac{d w_{r}}{2 \sqrt{2} \sigma_{r}},-a_{r}, a_{r}\right)}{a_{r}^{2}}
$$

where $a_{r}$ is a parameter obtained from the reflective area of the heliostat and the dispersion of the effective sunshape on the receiver, $l w_{r}$ and $d w_{r}$ are the height and the 
diameter of the receiver respectively and $\sigma_{r}$ is the dispersion of the effective sunshape on it.

\subsection{Atmospheric attenuation efficiency}

Atmospheric attenuation consists on energy losses derived from reflected radiation when traversing the air between every heliostat to the target receiver. This factor can get special relevance in SCRS whose heliostats are distributed over a vast surface because of the distance of the further ones to the receiver as well as in facilities placed in regions where visibility range is likely to change drastically by factors as pollution.

In this work, a simple model that assumes $40 \mathrm{~km}$ of visibility and depends only on the distance between a heliostat and the receiver, $d_{r e c}$, has been selected for calculating $\eta_{a a}$ according to [11] reasons

$$
\eta_{a a}= \begin{cases}0.99321-0.0001176 d_{r e c}+1.97 \cdot 10^{-8} d_{r e c}^{2} d_{r e c} \leq 1000 m \\ \exp \left(-0.0001106 d_{r e c}\right) & \text { otherwise }\end{cases}
$$

\subsection{Reflectivity efficiency}

The reflective surface of each heliostat can successfully redirect a certain percentage of total incident energy depending on its specifications and particular status (it could be damaged or specially dirty) independently of atmospheric attenuation and interception efficiency. At this work it is considered as constant for every heliostat.

\section{Parallelization approaches}

In general terms, optimizing any heliostat field will need numerous evaluations of the objective function depending on the optimizer and the quality of the results. Objective functions are usually time and/or memory demanding. Therefore, executing several field aptitude evaluations can make the optimization process, by extension, very difficult to handle or even unaffordable. In fact, it could not be optional when addressing extensive problems and the amount of memory needed has to be distributed between nodes.

In the case of using the above-detailed function described in Eq.(1) as the objective one, the memory should not be a problem for the common case, but its evaluation gets significantly time consuming in spite of the numerous simplifications that were made. The main reason is the computation of $\eta_{s b}$ which still needs to determine the orientation of the reflective plane of every heliostat and then analyze the set of lines generated from their discretized surfaces in relation with their potential shadowing/blocking neighbors at every studied instant.

Some optimization algorithms are specially suitable to be parallelized to improve their performance because of their nature and structure with interesting results (as in [14]). However, when possible, the scope can also be changed (maybe mixed) towards the objective function itself, with independence of the optimizer. This could be also useful when applying this kind of models in other tasks like automatic control. 


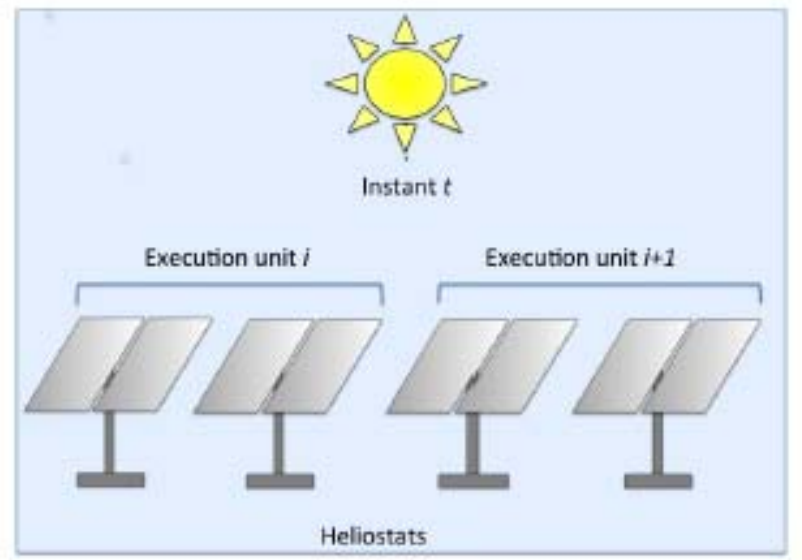

Fig. 1 Scheme of the parallelization by heliostats approach

For this selected case, Eq.(1) is perfectly suitable for being parallelized because of its internal configuration (computation of Eq.(2), (5) and sun positioning (3), (4) (with its associated timing)). In fact, in this work, three different parallel approaches considering distinct distributions have been considered.

\subsection{Parallelization by Heliostats}

When working with large set of elements, maybe the first and most natural way to accelerate the process is to divide that set into smaller parts that are associated to different execution units.

Talking about the computation of Eq.(1) in SCRS systems, the global set of heliostats can be also divided in blocks assigned to particular execution units (threads, processes... depending on the technology applied) that will compute the value of their specific region concurrently at every studied instant. For the problem at hand, an instant is a particular solar hour angle in a day that defines the apparent position of the Sun and, consequently, the whole heliostat field orientation. Each of these sub-values would be finally reduced to the global performance of the heliostat field in that particular studied instant. Furthermore, taking into consideration that there is no need to modify common values shared by every block (only reading general variables such as Sun position is needed), there are not critical sections to secure during per-block computing. This scheme is depicted in figure 1 . However, it must be noted that shadowing and blocking computation does not only depends on every heliostat itself but also of its shadowing and blocking candidates' orientation. Besides, the Sun position and solar irradiance are shared for the field in our model. Therefore, a barrier synchronization must be applied in order to set the global context of every particular time instant prior to start parallel computation per heliostat subsets.

This strategy is generally intuitive and not particularly difficult to implement. Besides, it should be applicable to numerous alternative and more complex functions because the theoretical context is easily generalizable: computations tend to be oriented to heliostats as their operation units. Furthermore, this kind of parallelization is valid to accelerate the process with independence of timing strategies. Then it would be valid even for a punctual evaluation when needed for specific objective (or maybe in a 


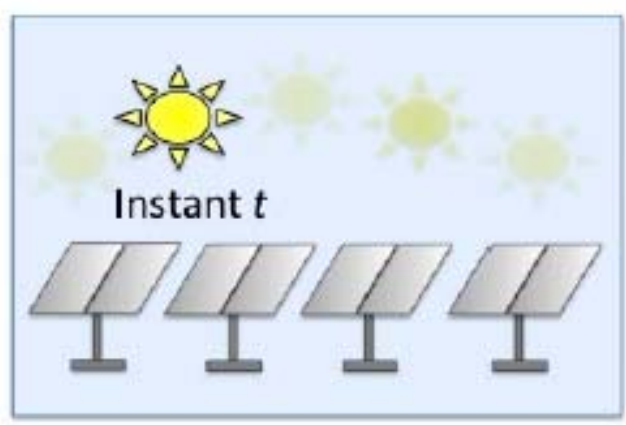

Execution unit $i$

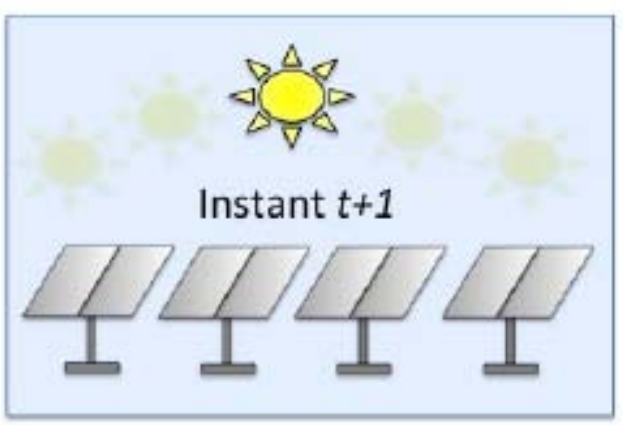

Execution unit $i+1$

Fig. 2 Scheme of the parallelization by instants approach.

simulation or control context): there is no need to count on a period to benefit from parallelization.

However, it is important to add that parallelization overhead is likely to be higher by applying this approach when working over periods of time. Additionally, it should be noted that computation linked to every heliostat has to be relevant (as in the example proposed). Finally, keeping the work load perfectly well balanced could be difficult: for this example, the further a heliostat is from the receiver the more impact is expected to have because of its larger number of potential blocking/shadowing neighbors.

\subsection{Parallelization by Instants}

When evaluating heliostat fields over a period of time as in Eq.(1), the process can be seen, from a higher point of view, as set of instants. This approach works over time instants instead of over heliostat subsets. In any case the idea is exactly the same: as every part of the problem can be treated independently (particular time instants) it is possible to perform calculations concurrently. This strategy, depicted in figure 2 , is also a natural option when heliostat fields are studied in more than a single instant. Therefore, it is also perfectly applicable in most optimization contexts whose objective function is related to energy production and optical efficiency along a certain period of time.

This approach is a coarser grain division than the previous one to the problem without any critical regions nor synchronizations between blocks (in spite of maintaining the obvious necessity of combining the result after computing all the blocks). Then, it is expected to reduce the parallelization overhead. Furthermore, in general terms instants should be easier to get well balanced because their load is less related to different distribution models and more linked to time.

On the other hand, it would require more memory than the previous one because it is necessary to create and maintain different context for the power station concurrently 

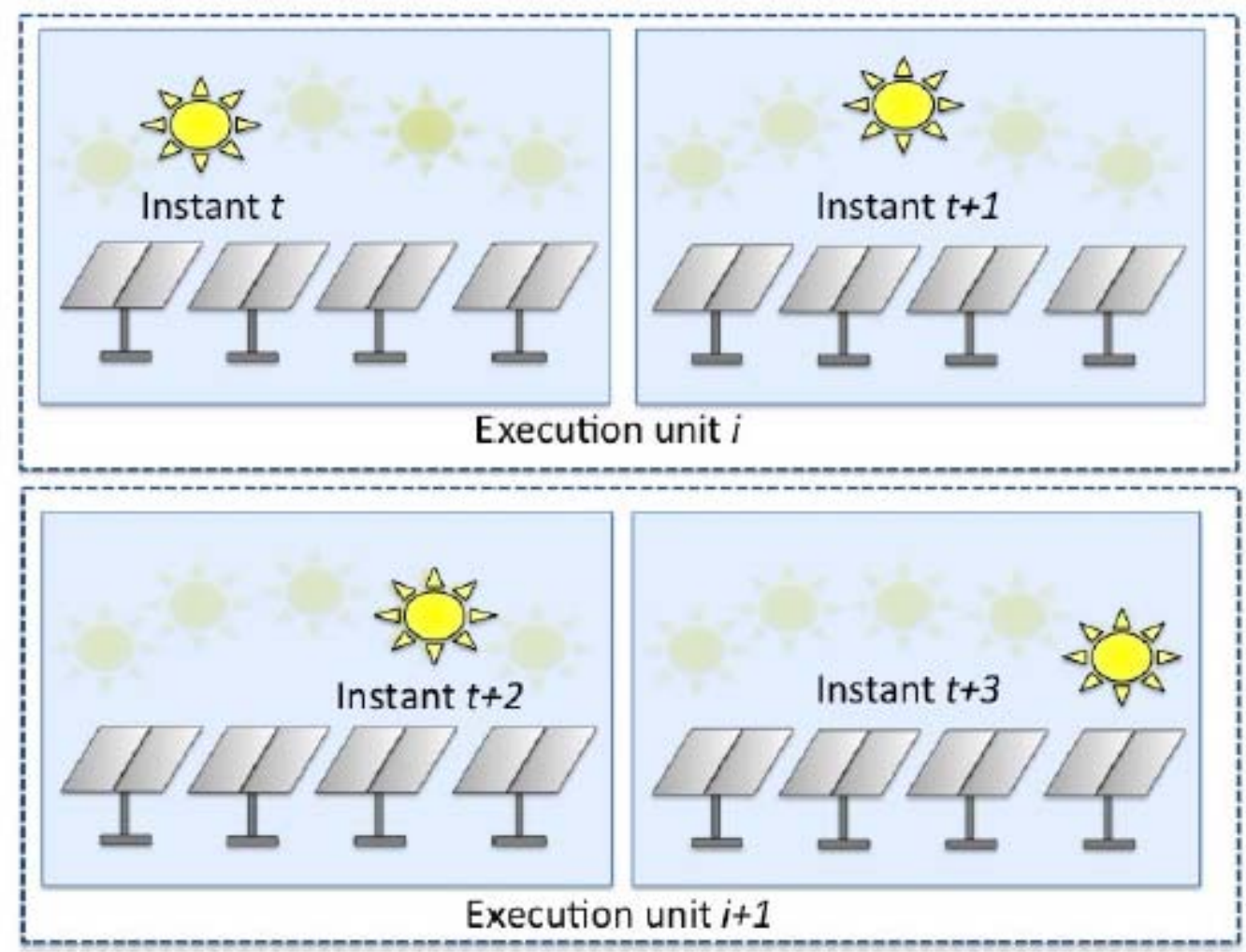

Fig. 3 Scheme of the parallelization by instant sets approach

(the specific apparent position of the Sun, the orientation of every heliostat, their particular potential shadowing neighbors, etc.). These points also make this strategy more difficult to be implemented. Furthermore, it is necessary to apply time discretization schemes and controlling that allow getting different time instants in advance. Finally, it is also important to note that this approach is only applicable when working on time periods as well as its perspective of the problem size and scalability depends on them.

\subsection{Parallelization by Instant Sets}

The natural evolution of the two previous exposed strategies is to consider working on a higher level blocking scheme to increment the grain size. Therefore, assigning blocks composed of different instants (as days) is a good way to extend the interesting points of the previous approach.

This third strategy, depicted in figure 3 , should reduce even more the parallelization overhead taking into account that less concurrence management is needed in execution time. Besides, stability and load balancing should be easier taking into account that it would be possible to operate with instants packs, as days, that would be very similar for the same periods of the year. Furthermore it is easier to grant that every divided block requires a considerable computing effort and the transition from the previous strategy should be quite direct (without increasing memory usage).

However, it must be noted the timing scheme applied should be modular enough to enable different independent instants generation. This approach also could suffer from lack of applicability when not working over long-term time periods. 


\section{Experimentation and results}

Function (1) has been implemented, as previously detailed, for experimentation purposes in four different ways: a pure sequential version and three basic parallelized versions (one per discussed strategy) using PThreads in $\mathrm{C}++$. The compilation is done with the '-O2' optimization flag in every case. The number of threads for parallel versions range from 2 to 12 . The testing platform is a cluster 'BullX' with 18 nodes Intel Xeon E5 2650 of 16 cores and 64 GB of shared RAM for each one. Experiments consist on registering the time required by the evaluation of performance of a particular SCRS power plant desing (with $\mathrm{Eq}(1)$ ) in a whole year for every implemented version. All the experiments has been repeated for five times to obtain an averaged result.

Optimization procedures should repeat this operation numerous times with different inputs in order to achieve good configurations. Therefore, an important speedup is expected with parallel versions in order to increase the evaluation possibilities in reasonable/feasible time.

The SCRS power plant is supposed to be in the equatorial region ( 0 degrees of latitude) with a tower that has a height of 100 meters with a cylindric receiver of 10.5 meters of vertical height and 8.4 meters of diameter over its peak. The number of heliostats range from 30 to 500 depending on the experiment. All of them are supposed to have a plain reflective surface of 100 square meters (in 10x10) whose central point is at 5 meters over the ground. They have an azimuth-elevation tracking system ([7]) with constant reflectivity.

Heliostats can be distributed over the available field in a wide range of different ways. In general terms, it is possible to work over a set of selected/discretized locations, to apply a distribution model based on a certain number of parameters that define the final layout or to mix both approaches when designing and optimizing heliostat fields. In this work, the number of available heliostats are distributed by the biomimetic layout proposed in [11] with the parameters obtained in [2]. This heuristic model, which gives very good results in numerous studies, is inspired from spiral patterns of the phyllotaxis disc (like florets on the head of sunflowers) and depends only on two optimization variables, $a$ and $b$, to define the layout. The required equations for generating the layout are:

$$
\begin{gathered}
\theta_{k}=2 \pi \varphi^{-2} k \\
r_{k}=a k^{b}
\end{gathered}
$$

where $k$ is the index of the heliostat, $\varphi$ is the golden ratio and $\theta_{k}$ are $r_{k}$ determines the azimuthal and radial distance respectively. The azimuth is measured clockwise from North direction while radial distance is taken as the separation from the heliostat to the tower over the land.

The average speedup $S$ obtained when applying the parallelization by heliostats is shown in figure 4 a). As previously introduced, the problem size varies from 30 to 500 studied heliostats and the generated threads for every instance of the problem are 2 , $4,6,8,10,12$ and 16 .

The obtained speedup is quite good taking into account that the load distribution implemented is very basic and that the expected overhead for this approach was significant. Results in figure 4 a) also shown good scalability because the speedup clearly increases for every instance when generating more threads (apart from the smallest one, with only 30 heliostats, where the obtained speedup does not even raise 4 with 12 
threads). However, in spite of the ascending behavior shown, the difference between the theoretical maximum and the obtained speedup also seems to increase slightly when deploying more threads. In any case, the evaluation time of function described in Eq.(1) is significantly reduced in all cases with this easily applicable strategy.

In relation to the second proposed approach, parallelizing by time instants, the speedup obtained under the same conditions is shown in figure $4 \mathrm{~b}$ ). In general terms, higher speedup is obtained when increasing both heliostat number and applied threads. This implies, by extension, a better overall efficiency. Scalability is also slightly improved attending to the behavior showed for large fields with more than 6 threads. Therefore, this approach is considered preferable than the previous one in terms of stability, speedup and efficiency (not as well in development effort) as expected.

Finally, the results obtained from the third parallelization strategy (dividing the problem in sets of different instants) in terms of speedup are shown in figure $4 \mathrm{c}$ ). It is important to mention that the size of every set is exactly of one day, what is not a balancing problem at 0 degrees of latitude, where days are quite regular along the year. As can be observed, the speedup is extremely regular in all cases as its linked to the long-term year study (what cannot be said when talking about the division by heliostats and the smaller instances). Therefore, there is not any problem where the parallelization performance is particularly bad and the overall speedup is quite good in general terms. However, it is in a midpoint between the division by heliostats (which behaves in a worse way in efficiency, speedup and stability) and the division by pure time instants (which seems to get slightly better speedup when increasing threads and heliostats in spite of being unstable with smaller instances).

\section{Conclusions and future work}

In this article, the problem of using parallel computing to obtain the aptitude of different heliostat field layouts is analyzed using a possible objective function as example. This general principle should not be seen only as a way to accelerate any optimization procedure (what, in any case, is really interesting and allow better search space explorations) but the key to afford problems whose objective function is so memory and cpu demanding that cannot be solved in traditional computing environments.

The function studied and implemented as a proof of concept has been parallelized in three different ways which are easily applicable for more complex situations in the same context. Empirical results from dividing the computation by heliostats, time instants and sets of them show that parallelizing is extremely useful to speedup the computation in any case. For instance, the calculation of the largest problem is reduced from 206 in sequential to approximately 15 with the best parallel approach. Division by time instants turns out to be the best choice for getting the maximum speedup in spite of its higher cost of implementation and memory requirements. Working over sets of instants is also a very interesting option for its stability and also its good overall performance. Finally, the strategy of dividing the problem by heliostat is not a bad option at all taking into consideration its implementation simplicity and the fact that it is oriented to large heliostat fields, the most common ones.

However, there are many other interesting points to work with in this area. First, it could be interesting to try mixed approaches like dividing the problem by instants linked to process that, at the same time, generate threads to compute subsets of heliostats. Second, it would be necessary to deeply study different load balancing schemes 

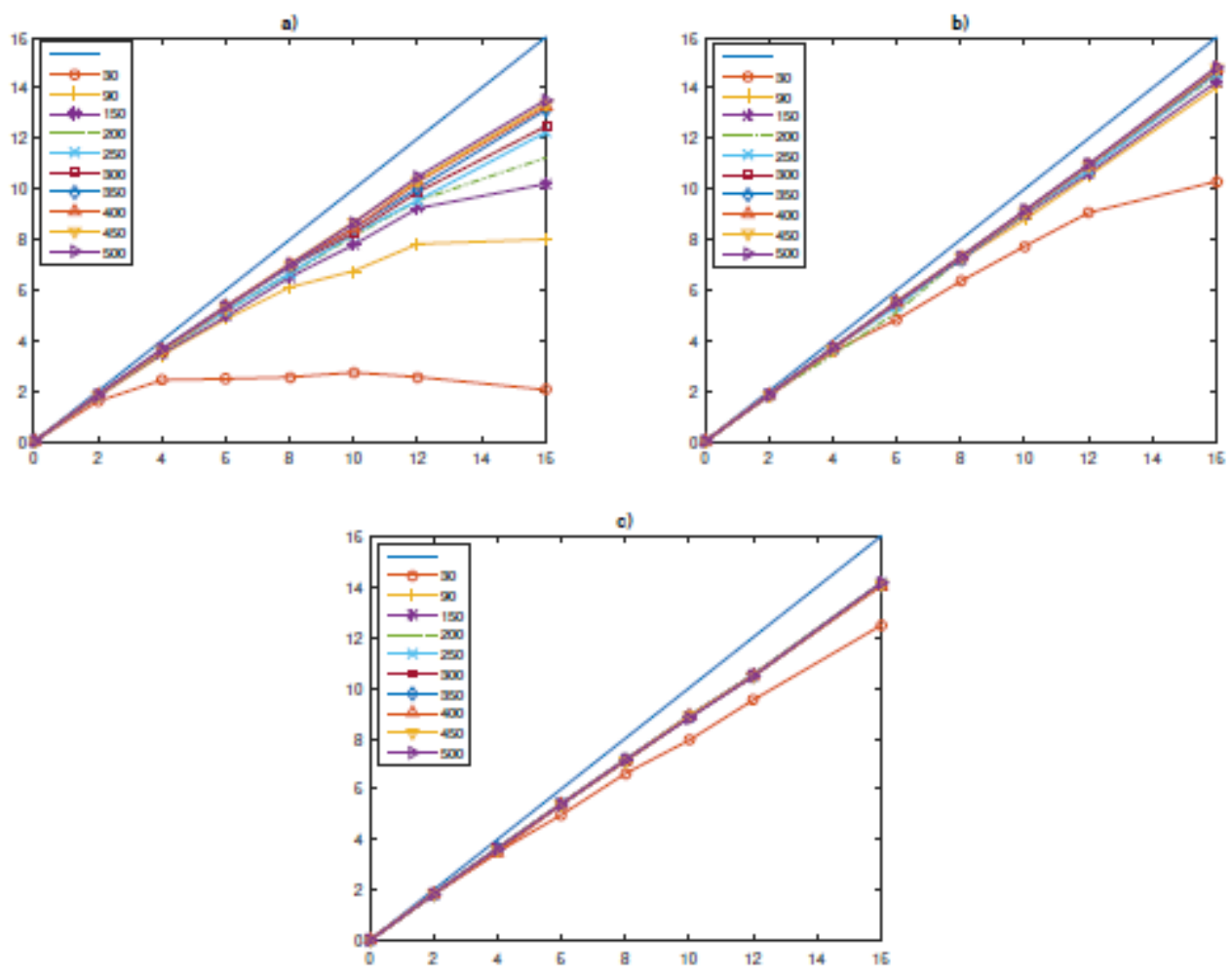

Fig. 4 Average speedup when parallelizing by sets of time instants a) heliostats b) time instants and c) sets of time instants.

in order to enhance obtained results. Another important point would be to implement and analyze these strategies by using MPI which is more portable, flexible and specially scalable to face extremely large problems being oriented to distributed memory systems. Finally, it could be useful to try parallelization techniques with more realistic and/or different functions as well as the real impact over their full optimization process. A possible second layer of parallelization applied to a chosen optimizer for this problem would be also a natural further development.

In any case, it is important to remark that functions and models applied to this specific problem can be intrinsically parallel instead of being just traditional and plain objective functions. Therefore, they can benefit from high performance computing techniques such as parallel programing in order to both speed up and improve optimization processes being able to analyze more candidate solutions per unit of time and make extremely large/complex problems affordable with independence of the optimizer particularities.

\section{References}

1. Behar, O., Khellaf, A., Mohammedi, K.: A review of studies on central receiver solar thermal power plants. Renewable and Sustainable Energy Reviews 23(0), 12-39 (2013)

2. Besarati, S., Goswami, D.: A computationally efficient method for the design of the heliostat field for solar power tower plant. Renewable Energy 69, 226-232 (2014)

3. Blanco-Muriel, M., Alarcón-Padilla, D., López-Moratalla, T., Lara-Coira M., j.v.n.p.y.p.: Computing the solar vector 
4. Camacho, E., Berenguel, M., Rubio, F., Martínez, D.: Control of solar energy systems. Springer Science \& Business Media (2012)

5. Cristóbal, A.: Diseño del campo de helióstatos para torres solares de receptor central. Degree Dissertation, Universidad Carlos III de Madrid, Spain (2011)

6. Ewert, M., Fuentes, O.: Modelling and simulation of a solar tower power plant

7. Geyer, M., Stine, W.: Power from the sun (powerfromthesun. net). JT Lyle Center (2001)

8. Kolb, G., Davenport, R., Gorman, D., Lumia, R., Thomas, R., Donnelly, M.: Heliostat cost reduction. In: ASME 2007 Energy Sustainability Conference, pp. 1077-1084. American Society of Mechanical Engineers (2007)

9. Laue, E.: The measurement of solar spectral irradiance at different terrestrial elevations. Solar Energy 13(1), 43-57 (1970)

10. Mustafa, M., Abdelhady, S., Elweteedy, A.: Analytical study of an innovated solar power tower (ps10) in aswan. International Journal of Energy Engineering 2(6), 273-278 (2012)

11. Noone, C., Torrilhon, M., Mitsos, A.: Heliostat field optimization: A new computationally efficient model and biomimetic layout. Solar Energy 86(2), 792-803 (2012)

12. Ramos, A., Ramos, F.: Strategies in tower solar power plant optimization. Solar Energy 86(9), 2536-2548 (2012)

13. Reddy, V., Kaushik, S., Ranjan, K., Tyagi, S.: State-of-the-art of solar thermal power plantsa review. Renewable and Sustainable Energy Reviews 27, 258-273 (2013)

14. Redondo, J., Fernandez, J., Garcia, I., Ortigosa, P.: Parallel algorithms for continuous competitive location problems. Optimisation Methods \& Software 23(5), 779-791 (2008)

15. Yao, Z., Wang, Z., Lu, Z., Wei, X.: Modeling and simulation of the pioneer $1 \mathrm{mw}$ solar thermal central receiver system in china. Renewable Energy 34(11), 2437-2446 (2009)

16. Zhang, H., Juchli, I., Favrat, D., Pelet, X.: Multi-objective thermoeconomic optimisation of the design of heliostat field of solar tower power plants. In: Engineering for Sustainable Energy in Developing Countries, Rio de Janeiro, Brasil (2007) 